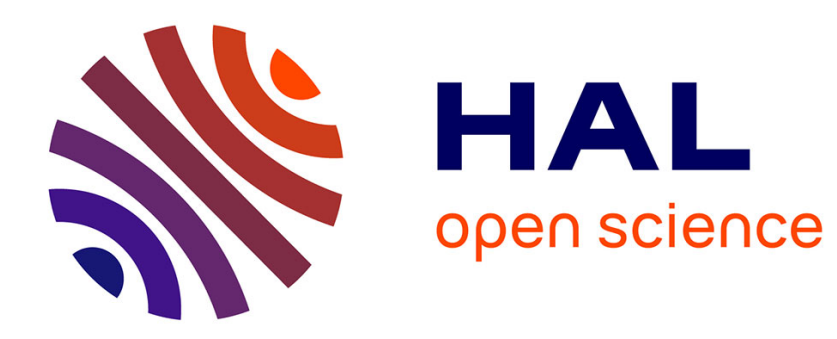

\title{
Le rôle de l'identité nationale dans le républicanisme critique
}

\author{
Sophie Guérard de Latour
}

\section{To cite this version:}

Sophie Guérard de Latour. Le rôle de l'identité nationale dans le républicanisme critique. Le Philosophoire, 2015, L'identité, 1 (43), pp.73-99. hal-02926233

\section{HAL Id: hal-02926233 \\ https://hal.science/hal-02926233}

Submitted on 2 Sep 2020

HAL is a multi-disciplinary open access archive for the deposit and dissemination of scientific research documents, whether they are published or not. The documents may come from teaching and research institutions in France or abroad, or from public or private research centers.
L'archive ouverte pluridisciplinaire HAL, est destinée au dépôt et à la diffusion de documents scientifiques de niveau recherche, publiés ou non, émanant des établissements d'enseignement et de recherche français ou étrangers, des laboratoires publics ou privés. 


\title{
LE RÔLE DE L'IDENTITÉ NATIONALE DANS LE RÉPUBLICANISME CRITIQUE
}

\author{
Sophie Guérard de Latour
}

Vrin | «e Philosophoire »

2015/1 n 43 | pages 73 à 99

ISSN 1283-7091

ISBN 9782353380466

Article disponible en ligne à l'adresse :

https://www.cairn.info/revue-le-philosophoire-2015-1-page-73.htm

Distribution électronique Cairn.info pour Vrin.

(C) Vrin. Tous droits réservés pour tous pays.

La reproduction ou représentation de cet article, notamment par photocopie, n'est autorisée que dans les limites des conditions générales d'utilisation du site ou, le cas échéant, des conditions générales de la licence souscrite par votre établissement. Toute autre reproduction ou représentation, en tout ou partie, sous quelque forme et de quelque manière que ce soit, est interdite sauf accord préalable et écrit de l'éditeur, en dehors des cas prévus par la législation en vigueur en France. Il est précisé que son stockage dans une base de données est également interdit. 


\title{
Le rôle de l'identité nationale dans le républicanisme critique
}

\section{Sophie Guérard de Latour}

\begin{abstract}
Résumé
Contre les récupérations idéologiques du thème de « l'identité nationale », ce texte mobilise la perspective du néo-républicanisme afin de réhabiliter la portée philosophique de cette notion controversée. Le concept de non-domination y opère comme un vecteur critique susceptible d'articuler l'identification à une communauté politique et l'intégration des minorités ethnoculturelles. Sur cette base philosophique, le texte défend la légitimité d'une éducation qui inculque aux futurs citoyens une vision multiculturelle de leur identité nationale.

\section{Abstract}

This article draws on the perspective of neo-republicanism to reassess the legitimacy of the notion of «national identity », against its conservative and xenophobic politicization. The concept of non-domination operates as a critical tool to articulate the process of citizens'identification to a political community and the integration of ethno-cultural minorities. This philosophical basis allows to plead for an education that equip future citizens with a multicultural picture of their national identity.
\end{abstract}

\section{Introduction}

La création du Ministère de l'Immigration et de l'Identité nationale en mai 2007 par le président Nicolas Sarkozy a profondément discrédité

1. Je tiens à remercier Nael Desaldeleer, Luc Foisneau, Charles Girard, Christopher Hamel, Juliette Roussin et Mathilde Unger de m'avoir aidé à préciser l'argument de ce texte par leurs remarques éclairantes. 
l'expression d'《identité nationale ». Bien que la communauté nationale soit la base même de la légitimité républicaine, la volonté politique d'en institutionnaliser le contenu identitaire a été largement dénoncée comme une dérive nationaliste, que certains ont été jusqu'à qualifier de « xénophobie d'État » ou « de gouvernement ${ }^{2}$ ».

D'un point de vue strictement politique, on peut en effet interpréter l'annonce de la création d'un tel ministère comme une stratégie de séduction dirigée vers l'électorat frontiste, dont elle a repris les thèmes fondateurs en avalisant officiellement la thèse selon laquelle l'immigration représente une menace, un "problème " pour la nation française (non seulement les immigrés qui souhaiteraient s'installer en France, mais aussi ceux qui y vivent déjà de façon durable). Une telle stratégie de cannibalisation a été couronnée de succès puisque, comme nombre de commentateurs l'ont souligné, c'est cette promesse électorale qui a donné l'avantage à Nicolas Sarkozy contre Ségolène Royal dans la course au pouvoir présidentiel ${ }^{3}$.

L'efficacité de cette stratégie repose en partie sur la diversion qu'elle rend possible, puisqu'en détournant l'attention des problèmes relatifs aux inégalités sociales, elle permet de rassembler des classes sociales que leurs intérêts économiques séparent. L'instrumentalisation politique de l'identité nationale s'inscrirait à ce titre dans la vieille logique du bouc émissaire qui cherche à préserver la cohésion du groupe social en mettant à l'index la personne ou le groupe jugés responsables de tous les maux. Elle ajoute à cela une dimension idéologique au sens marxiste du terme, puisqu'elle offre aux élites un moyen efficace de donner du sens aux difficultés que vivent les classes populaires, tout en neutralisant leurs revendications de justice sociale. L'analyse historique des débats politiques indique ainsi que depuis la fin du XIX ${ }^{\mathrm{e}}$ siècle, le thème de l'identité nationale a été tout particulièrement exploité par les partis de droite. Les contraintes structurelles liées à la « démocratie du public », où se combinent les effets de la marchandisation de la sphère publique et les limites imposées aux discours publics par les valeurs démocratiques, ont contribué à faire de la «nation » un argument à la

2. Sous la direction de Jérôme Valluy, « Xénophobie de gouvernement, nationalisme d'État », Cultures \& Conflits, $\mathrm{n}^{\circ}$ 69, printemps 2008.

3. G. Noiriel, À quoi sert « l'identité nationale», Paris, Agone, 2007, p. 81 et sq. 
fois recevable et efficace pour faire passer des politiques conservatrices et anti-égalitaires ${ }^{4}$.

L'épisode du Ministère de l'Immigration et de l'Identité nationale invite donc à la plus grande prudence quand on évoque la question de l'identité nationale. Pourtant, l'identité nationale ne peut pas faire l'objet d'une analyse strictement fonctionnaliste/stratégique. Que ce thème tende à être perverti par la démocratie du public en "élément de langage » conservateur ne le prive pas de toute portée normative. Il faut souligner ici l'écart qui sépare la pratique politique des analyses théoriques. Du point de vue de la théorie politique, en effet, la nation joue un rôle clé quand il s'agit de définir la philosophie républicaine et sa façon de penser la légitimité démocratique. Comme on le verra par la suite, le républicanisme entend se distinguer à cet égard du libéralisme contemporain, du fait de la conception davantage communautarienne qu'il adopte des liens politiques: la liberté, pour les républicains, ne se limite pas aux droits de l'individu. Elle se conjugue pour ainsi dire au singulier, puisque c'est le peuple qui est libre, en tant qu'il affirme sa volonté de décider souverainement de son destin. Mais, un peuple se distingue des autres par l'identité nationale qui le caractérise en tant que collectivité historique et culturelle singulière. L'instrumentalisation politique de l'identité nationale place dès lors les républicains devant le dilemme suivant : admettre que la République n'est rien sans la nation et se lancer sur le terrain glissant des discours identitaires, au risque de devenir l'allié objectif des nationalistes qui détournent l'esprit républicain de sa visée universaliste ; ou bien refuser de s'engager dans ce type de débat pour en éviter les pièges mais se résigner à voir l'une des bases de la philosophie républicaine être sapée par l'opportunisme politique.

Dans ce qui suit, je voudrais me pencher sur la position du républicanisme critique afin d'ouvrir une voie pour dépasser ce dilemme. Le républicanisme critique, tel que Cécile Laborde le définit dans ses textes ${ }^{5}$, propose une « refondation de l'idéal de la citoyenneté républicaine $»^{6}$, en procédant à la critique du républicanisme français, «à

4. G. Noiriel, À quoi sert "l'identité nationale », op. cit.; Immigration, antisémitisme et racisme en France. Discours publics, humiliations privées (XIXe-XXe siècles), Paris, Fayard, 2007.

5. C. Laborde, Critical Republicanism. The Hijab Controversy and Political Theory, Oxford, Oxford University Press, 2008 ; Français, encore un effort pour être républicains !, Paris, Seuil, 2010.

6. C. Laborde, Français, encore un effort pour être républicains !, op. cit., p. 9. 
la lumière de la critique sociale et de la philosophie néo-républicaine de la non-domination ${ }^{7}$. Les néo-républicains considèrent, à la suite de Philip Pettit ${ }^{8}$, que la liberté ne se limite pas à la non-interférence mais qu'elle exige la non-domination. Ce que les lois doivent neutraliser ne réside pas tant dans les interférences effectives d'autrui dans la sphère d'action de chacun, mais plus largement les situations de domination que créent les rapports de dépendance et de pouvoir entre les individus, soumettant ces derniers à la possibilité d'interférences arbitraires (c'est-à-dire de décisions qui ne reflètent pas leurs propres volontés ou intérêts). Toutefois, tout en élargissant la conception des interférences illégitimes par rapport à la tradition libérale, les néo-républicains restent proches de cette tradition en ce qu'ils maintiennent une conception négative de la liberté, laquelle ne prétend pas imposer de conception substantielle de la vie bonne. En opérant un retour critique sur le républicanisme français à partir des positions néo-républicaines, Laborde espère ainsi apporter un correctif libéral à certaines tendances du républicanisme français, notamment en envisageant d'un œil plus favorable que les «républicains classiques ${ }^{9}$ la question des revendications minoritaires (minorités religieuses, ethniques ou raciales). Ce correctif ne prétend pas pour autant réduire la spécificité de l'idéal républicain par rapport au libéralisme contemporain; il cherche au contraire à mettre en lumière l'originalité de ces principes et des solutions politiques qu'il propose en matière de pluralisme religieux et culturel. Mais une telle entreprise se heurte à la difficulté suivante : dans quelle mesure le républicanisme critique reste-t-il républicain si la critique qui structure sa refondation est d'inspiration libérale ? Ou, pour le dire autrement, dans quelle mesure le républicanisme critique offret-il une alternative réellement originale au libéralisme contemporain, à partir du moment où il en accepte les postulats de base (et notamment le primat de la liberté négative sur la liberté positive) ?

7. Ibidem, p. 8.

8. P. Pettit, Républicanisme. Une théorie de la liberté et du gouvernement, Paris, Gallimard, 1997.

9. Laborde a choisi cette expression pour traduire celle d'official republicans utilisée dans Critical Republicanism. Les républicains classiques renvoient non pas à un " groupe d'individus identifiable », ni à une « position sociologique », mais plutôt à « des logiques argumentatives », unifiées et apparemment cohérentes, qui structurent les débats politiques français et s'inscrivent globalement dans la tradition du républicanisme de la III ${ }^{\mathrm{e}}$ République (voir Français !, op. cit., p. 11-12) 
Je soutiendrai que le républicanisme critique parvient à suivre la voie étroite d'un républicanisme rendu hospitalier aux différences sans verser pour autant dans le libéralisme. La façon dont le républicanisme critique s'approprie la question de l'identité nationale m'offrira l'occasion d'analyser en détail cette possibilité. Je commencerai par rappeler les raisons pour lesquelles les néo-républicains accordent, avec les républicains classiques, une valeur normative particulière à l'identité nationale. Je préciserai ensuite, en exploitant le potentiel critique du concept de non-domination, propre au néo-républicanisme, comment cette philosophie politique permet de repenser l'identité nationale en des termes inclusifs. Je terminerai en soutenant que cette perspective critique invite à aller plus loin que les théories du nationalisme libéral et que les justifications instrumentales de l'identité nationale que ces théories offrent généralement.

\section{Première partie : Liberté républicaine et identité nationale}

Si la question de l'identité nationale reste un enjeu crucial de la théorie politique, malgré les dérives auxquelles elle conduit dans la vie politique, c'est que la nation est l'un des fondements essentiels de la tradition de pensée républicaine.

Cette tradition envisage la politique comme « chose publique », dans la mesure où elle concerne non pas une collection d'individus privés, mais un peuple souverain, c'est-à-dire un ensemble de citoyens qui se perçoivent comme engagés dans un destin commun et qui sont soucieux de le maîtriser. Le «peuple» ou la « nation » sont donc la source de la souveraineté politique.

Il est important de souligner ici le rapport essentiel qui unit les concepts de « peuple » et de liberté en revenant sur l'ontologie communautarienne qui fonde le républicanisme. Bien que la tradition républicaine, dans ses sources romaines, soit autant attachée à l'indépendance des individus que la tradition libérale, la première ne pense pas la liberté individuelle dans les mêmes termes que la seconde. Les libéraux, comme Locke ou Kant, envisagent la liberté comme une sorte de puissance naturelle qui détermine à la fois le rôle de l'État (celui de garantir les droits naturels) et qui en fixe les limites (celles de ne pas empiéter sur ces droits inaliénables). À l'inverse, comme l'ont montré 
Quentin Skinner ${ }^{10}$ et Philip Pettit, la tradition républicaine, depuis Cicéron jusqu'à Rousseau, dérive la liberté du citoyen non d'un état naturel, mais du statut politique qui la rend possible : « la citoyenneté est un statut qui n'existe qu'en référence à un état de droit légitime ${ }^{11}$.

Il en résulte une conception radicalement différente des lois, puisque celles-ci ne sont plus perçues comme les contraintes imposées à la liberté de l'homme naturel, indispensables à sa préservation dans le contexte social, mais comme les institutions créatrices de la liberté individuelle. Les républicains ne n'établissent donc aucun rapport exclusif entre les lois et la liberté et insistent au contraire sur leur co-originarité. Dans sa défense contemporaine du républicanisme, Pettit a reformulé cette divergence dans les termes de l'opposition entre option-freedom et agency-freedom ${ }^{12}$. Alors que la première conception met l'accent sur les fins de l'action, sur la gamme de choix que l'individu trouve à sa disposition, la seconde est tournée vers la qualité des rapports intersubjectifs qui conditionnent la capacité même de choisir. La liberté suppose en effet qu'un individu se voit reconnaître, formellement et symboliquement, le statut d'agent capable de s'autodéterminer. Les républicains ont traditionnellement mis l'accent sur cette forme de liberté, en insistant sur l'importance de l'ethos égalitaire qui fonde la légitimité politique : les rapports de domination ne peuvent être neutralisés qu'à condition que les lois confortent chez les citoyens le sentiment de leur égale valeur et qu'elles découragent réciproquement les comportements serviles, lesquels sont les principaux vecteurs des relations de dépendance arbitraires (i.e. de la domination). C'est la raison pour laquelle les républicains refusent de distinguer la question de la justice politique de celle de la constitution de la communauté politique : il faut commencer, comme y insiste Rousseau au début du Contrat social, par « examiner par quel acte un peuple est un peuple ${ }^{13}$ si l'on veut saisir la véritable nature de la liberté.

Cette ontologie communautarienne place les républicains devant la nécessité de se pencher sérieusement sur la question de l'identité du

10. Q. Skinner, La liberté avant le libéralisme, Paris, Seuil, « Liber », 2000.

11. P. Pettit, Républicanisme, op. cit., p. 58.

12. P. Pettit, «Option-Freedom and Agency-Freedom», Journal of Theoretical Politics, 15(4), 2003, p. 387-403.

13. Voir Rousseau, «Avant donc que d'examiner l'acte par lequel un peuple élit un roi, il est bon d'examiner l'acte par lequel un peuple est un peuple » (J-J Rousseau, Contrat social, Paris, G.-F., 1996, I, 3, p. 50, nous soulignons) 
peuple, dans la mesure où leur conception de la liberté est étroitement dépendante de l'appartenance à une communauté de citoyens ${ }^{14}$. On fera remarquer ici que, de façon assez surprenante, ce sont plutôt des penseurs libéraux, comme Yael Tamir ou Will Kymlicka, qui ont lancé cette réflexion ${ }^{15}$. Tous deux ont souligné que la portée universelle du projet civique n'a pas fait disparaître la diversité culturelle des groupes nationaux; les nations restent des communautés éthiques, attachées à une certaine identité politique qui n'a pas la portée universelle des valeurs politiques qu'elles professent et qui justifient leur séparation territoriale. Pourtant, bien que ces auteurs aient avancé des arguments importants pour réconcilier l'attachement aux cultures nationales avec les principes de la démocratie libérale, ils restent ancrés dans un schéma de pensée libéral qui envisage la liberté en termes de choix et d'opportunités. Il reste donc à cerner plus clairement ce que la perspective républicaine apporte à ce débat.

Chez les républicains, la question de l'identification collective au «peuple» a été traditionnellement envisagée comme un processus d'adhésion à des valeurs et à des institutions politiques ${ }^{16}$. C'est ce qu'on retrouve dans le thème classique du patriotisme, où l'amour de la patrie découle logiquement du caractère statutaire de la liberté, à savoir du fait que la liberté du citoyen dépend de son appartenance à une cité. Comme le souligne Pettit, dans un passage où il défend l'actualité de la vertu de patriotisme, «si nous chérissons notre propre citoyenneté et notre propre liberté, nous devons chérir dans le même temps le corps social auquel il faut appartenir pour se voir reconnaître ce statut. $\gg{ }^{17}$.

Or, il faut bien admettre que la synthèse opérée entre les valeurs civiques et l'identité collective dans la version traditionnelle du patriotisme ne saurait être transposée telle quelle dans le contexte des démocraties modernes, à cause du changement profond de nature des liens politiques.

- D'abord, le changement d'échelle entre les cités antiques et les États-nations modernes empêche de faire une lecture strictement

14. S. Guérard de Latour, "Reworking the neo-republican sense of belonging ", Diacritica, 24/2, 2010, p. 85-105.

15. Y. Tamir, Liberal Nationalism, Princeton, Princeton University Press, 1990 ; W. Kymlicka, La citoyenneté multiculturelle. Une défense libérale du droit des minorités, Paris, La Découverte, 1995.

16. M. Viroli, For the Love of Country. Essays on Patriotism and Nationalism, Oxford, Oxford University Press, 1997.

17. P. Pettit, Républicanisme, op. cit., p. 348. 
civique de l'identité collective des citoyens modernes. L'identification à l'œuvre dans le patriotisme des Anciens était facilitée par les rapports d'interconnaissances que l'espace de vie offert par la cité rendait possible. Le caractère collectif de la liberté s'incarnait alors dans les traits familiers des visages des compatriotes. L'attachement de tous aux institutions de la cité était matérialisé par la pratique commune et quotidienne de la démocratie directe. En revanche, à l'ère des États-nations modernes, où la population se compte en millions d'individus et où le peuple délègue à ses représentants le soin de le gouverner, l'anonymat et la distance deviennent la norme des relations entre compatriotes, ce qui modifie en profondeur le processus de leur identification collective. Les travaux menés à partir des années 1980 sur les processus de construction nationale ont montré comment le nationalisme s'est alors substitué au patriotisme, et par quels moyens les politiques d'assimilation culturelle orchestrées par l'État central ont permis de tracer les frontières d'une « communauté imaginée ${ }^{18}$. Ce champ de recherches a contribué à brouiller la distinction généralement reçue entre « nation civique » et « nation ethnique » : si le peuple désigne en théorie, dans les démocraties modernes, la source de la légitimité politique - en tant que communauté de citoyens unis par des principes communs - il renvoie, dans la réalité historique et sociologique de ces démocraties, à une nation, c'est-à-dire à un groupe historique et culturel particulier. Dès lors, la formation des nations modernes ne relève pas seulement de l'affirmation d'un idéal civique, elle participe aussi d'une forme culturelle de nationalisme qui cherche à ancrer l'identité collective dans « une personnalité collective primordiale (la nation) disposant d'un nom, d'origines uniques, d'une histoire, d'une culture, d'un territoire, et de pratiques sociales et politiques ${ }^{19}$. Or, étant données d'une part l'extension du territoire national, qui intègre souvent des minorités nationales marginalisées par la culture officielle, et d'autre part la présence de citoyens issus de l'immigration qui ne sont plus systématiquement exclus de la vie démocratique (à l'instar des métèques dans le régime athénien), la capacité de l'identité politique à être culturellement ouverte et attractive pour ces groupes minoritaires devient problématique. Comment se sentir patriote, en effet, dans une démocratie qui

18. B. Anderson, L'imaginaire national, Paris, La Découverte, 2002.

19. J. Hutchinson, «Re-interpreting Cultural Nationalism », Australian Journal of Politics and History, 45/3, 1999, p. 394. 
officialise une identité nationale non représentative de la diversité des origines culturelles de chaque citoyen ?

- Ensuite, la modernité oblige à composer avec la désacralisation de l'activité politique. On sait que la vertu du patriotisme prenait sens dans un contexte culturel marqué par la dignité éminente de la vie politique. La capacité du citoyen à se sacrifier pour défendre sa patrie était l'expression suprême des vertus civiques qui devaient au quotidien l'inciter à faire passer les affaires publiques avant ses intérêts privés. Or, la modernisation économique et politique des sociétés occidentales a progressivement démagnétisé l'idéal du citoyen vertueux pour le remplacer par celui de l'individu en quête de bien-être et soucieux de préserver ses libertés face à la puissance grandissante de l'État moderne. Là encore, la question qui se pose est celle du pouvoir de séduction de l'identité commune : comment l'identification à la nation peut-elle rester forte et capable de nourrir le sens communautaire à une époque où la participation et l'engagement civique ne sont plus les types de comportement structurant la vie sociale?

Il est intéressant de relever qu'aucune de ces deux difficultés n'a conduit les républicains contemporains - aussi bien du côté des néorépublicains anglo-saxons que des républicains classiques français - à minimiser le rôle de l'identification à la nation dans la constitution de la légitimité politique.

En ce qui concerne le premier point, les républicains classiques n'ont pas jugé les critiques multiculturelles suffisamment fortes pour invalider le principe de l'intégration à une culture nationale. La nation, souligne Dominique Schnapper, n'est pas une ethnie parmi d'autres. Bien que l'identité qu'elle désigne comporte une part d'ethnicité liée à son contexte historique, elle reste structurée par le "projet» de la citoyenneté moderne et repose de la sorte sur un "principe d'inclusion potentielle ${ }^{20}$. L'ethnicité, au contraire, obéit à une logique de pureté qui est intrinsèquement traditionaliste et exclusive. Se réclamant des positions anti-racialistes des premiers sociologues, et notamment d'Émile Durkheim 21, Schnapper défend la spécificité d'une intégration sociale qui, loin d'encourager la reproduction des similitudes repose sur l'affirmation des différences et à la promotion de la personnalité

20. D. Schnapper, La relation à l'autre. Au cour de la pensée sociologique, Paris, Gallimard, 1998, p. 445.

21. Ibid., op. cit., p. 397. 
de chacun, grâce aux droits individuels garantis par l'État. En termes durkheimiens, de la " solidarité mécanique » propre aux sociétés traditionnelles à la "solidarité organique », caractéristique des sociétés modernes, la différence n'est pas de degré mais de nature, dans la mesure où la seconde repose sur un type spécifique de lien social susceptible de combiner l'attachement à la communauté nationale et l'ouverture à la diversité des origines et des appartenances. Schnapper en conclut qu'il est donc légitime de chercher à intégrer l'ensemble des citoyens à la culture nationale, même si cette dernière reflète inévitablement un certain héritage historique et culturel, car tel est «le prix à payer pour que tous les citoyens participent pleinement à la société nationale ${ }^{22}$. La défense d'une culture nationale ne doit donc pas être systématiquement confondue avec une forme d'ethno-nationalisme. Comme Vincent Martigny le souligne dans son analyse du nationalisme culturel français, le processus d'homogénéisation culturelle encouragé par les républicains après la Révolution française n'était pas caractérisé par la promotion d'une identité ethnique particulière, celle de Paris ou de l'île de France, il s'est au contraire tout autant appuyé sur l'exaltation des « petites patries » que sur la culture universaliste du centre du pays ${ }^{23}$.

Le maintien du principe de l'intégration nationale se retrouve dans la position du républicanisme critique. Laborbe souligne ainsi le rapport strictement instrumental que le «modèle d'intégration républicain » établit entre l'affinité culturelle et l'appartenance politique : loin de tirer profit de la puissance d'État pour imposer une culture au nom de sa valeur intrinsèque, ce modèle met l'identité nationale au service de l'objectif politique qui consiste à créer une communauté de citoyens libres $^{24}$. Un tel projet prolonge l'intuition des fondateurs du républicanisme à propos du patriotisme, à savoir que la culture nationale est le lien civique indispensable à la constitution du peuple et à la réalité du principe d'autogouvernement.

En ce qui concerne le second point, relatif au discrédit de la vertu de patriotisme, on trouve aussi dans le néo-républicanisme dont s'inspire le républicanisme critique un argument intéressant pour défendre son actualité à partir du lien observable entre « intériorisation et iden-

22. D. Schnapper, La relation à l'autre, op. cit., p. 445.

23. V. Martigny, "The Importance of Culture in Civic Nations: Culture and the Republic in France », Studies in Ethnicity and Nationalism, vol. 8, n 3, 2008, p. 554.

24. C. Laborde, Critical Republicanism, op. cit., p. 178. 
tification ». Sans revenir au modèle antique du citoyen engagé, Pettit prolonge malgré tout la tradition républicaine en affirmant que la promotion de la liberté ne dépend pas seulement des bonnes lois mais qu'elle suppose aussi de bonnes mœurs. Promouvoir la non-domination exige à ce titre de la « civilité », c'est-à-dire une certaine propension chez les citoyens à agir d'une façon qui n'encourage pas les rapports de domination. On considère généralement que l'égoïsme des individus modernes compromet leur capacité à développer ce genre de disposition altruiste. Mais ce préjugé, estime Pettit, procède d'une vision partielle de la civilité qui néglige le fait que les individus intériorisent certaines normes morales ou politiques en s'identifiant aux groupes dont les intérêts sont liés à ces valeurs. Dès lors, "cette image tient compte des efforts de contrainte sur soi qu'exige la réalité de la civilité, mais elle ne tient pas compte des coûts que représenterait sa non-réalisation pour l'identité du moi ${ }^{25}$. Rappeler le rôle que joue l'identification dans l'intériorisation des normes permet a contrario de réhabiliter en partie le sens de la vertu, en évitant l'objection de l'héroïsme moral (lequel ne semble plus pouvoir être exigé des individus modernes). L'identification par laquelle « l'agent laisse pour ainsi dire dériver son moi personnel (pour permettre) à une identité différente (...) de remplacer celle qui y était attachée » n'est pas un processus d'ordre intentionnel, purement instrumental ; « c'est quelque chose qui advient aux individus aussi naturellement que la respiration ${ }^{26}$. C'est donc spontanément que nous sommes amenés à nous engager en faveur du groupe auquel nous sommes liés par certaines valeurs, notamment par des valeurs républicaines comme celle de la non-domination. Le cas de cette valeur politique est toutefois particulier : « il s'agit en effet d'un bien commun, dans la mesure où aucun membre d'un groupe vulnérable - les femmes, les Noirs, et en dernier ressort, les membres de la société dans son ensemble - ne peut espérer en jouir pleinement sans que tous les membres du groupe en jouissent également ${ }^{27}$.

Ce second point complète le premier argument sur le caractère instrumental de l'intégration culturelle dans le républicanisme: pour les néo-républicains, il est légitime de promouvoir l'attachement des citoyens à leur identité nationale, dans la mesure où cette identité partagée conditionne la réalisation de leur projet politique ; c'est la soli-

25. P. Pettit, Républicanisme, op. cit., p. 345.

26. Ibid., p. 346.

27. Ibid., p. 347. 
darité créée entre eux par le sentiment d'appartenir à une même nation qui les engage spontanément à refuser et à combattre les rapports de domination.

\section{Deuxième partie : Contestation de la domination sociale et pluralisation de l'identité nationale}

Ce premier moment de la réflexion a permis de préciser les raisons pour lesquelles une approche strictement fonctionnelle de l'identité nationale ne saurait suffire. La nation reste un principe normatif clé du républicanisme et les types de récupération et d'instrumentalisation dont elle fait l'objet dans les pratiques politiciennes n'invalident pas ce principe en tant que tel. Toutefois, revenir aux fondamentaux du républicanisme pour préciser ce point $\mathrm{m}$ 'a conduit à mettre davantage l'accent sur ce qui rapproche les républicains classiques et les néo-républicains que sur ce qui les sépare. Il s'agit à présent d'éclairer les points de rupture entre ces deux versions de républicanisme, en montrant comment la base conceptuelle du néo-républicanisme offre un angle critique sur la question de l'intégration nationale qui manque au républicanisme classique. On peut reprocher en effet à ce dernier de ne pas être suffisamment attentif aux effets de domination que produit le nationalisme culturel : même si ce dernier ne se confond pas avec le nationalisme ethnique, il reste susceptible d'imposer une conception biaisée de l'identité nationale qui conforte des rapports d'inégalité entre les citoyens. À l'inverse, le néo-républicanisme, par la redéfinition qu'il propose de la liberté, semble mieux à même de les prévenir.

Fonder la liberté républicaine sur le concept de la non-domination engage, comme on l'a vu, une certaine qualité des rapports entre citoyens qui relève à la fois des normes qu'ils ont intériorisées et de l'identité collective à laquelle ils associent ces normes. Il en résulte immédiatement que la manière dont l'identité collective est construite influence l'effectivité des normes partagées. Comme le souligne Laborde, «dans une conception intersubjective de la citoyenneté, une personne peut jouir formellement des droits civiques tout en se étant exclue de la représentation imaginaire de la patrie, à cause de la façon dont son identité est perçue ou lui est assignée. Ainsi, d'un point de vue 
républicain, le statut civique des gens peut pâtir de la façon dont les discours construisent l'identité collective ${ }^{28}$.

Cette situation dans laquelle l'égalité civique devient formelle, faute des conditions culturelles d'identification à la nation, est typiquement celle des immigrés vivant en France et de leurs descendants. Même s'il est bien connu que l'immigration a joué un rôle démographique, économique et social fondamental dans l'émergence de la France contemporaine, la France n'est pas considérée comme un pays d'immigration, à l'instar des États-Unis, du Canada ou de l'Australie. L'immigration souffre en France, comme l'a montré Gérard Noiriel, d'un «non-lieu de mémoire $»^{29}$ : aucun lieu à forte charge symbolique ne permet d'installer dans l'imaginaire national la «place légitime» des immigrés et de leurs descendants, ce qui encourage d'après Noiriel un «processus d'extériorisation $»^{30}$ des immigrés par rapport à la communauté nationale. Pour analyser les ressorts de ce processus, l'historien a suivi deux voies différentes. Il s'est d'abord intéressé à «l'obstacle épistémologique » qui explique pourquoi l'immigration française a pendant longtemps été négligée par la discipline historique. Cela l'a conduit à opérer la généalogie des discours produits par les savants républicains sur la «nationalité française », de Michelet à Braudel, en passant par Renan et Vidal de la Blache: Noiriel a ainsi dégagé «l'a priori de l'enracinement » que ces discours ont progressivement construit, en fondant l'identité française sur un héritage ancestral qui auraient fixé des cadres culturels bien avant la révolution industrielle, dans lesquels les immigrés se seraient fondus sans en modifier la substance ${ }^{31}$. Cet a priori explique en partie comment une expression telle que «Français de souche », formulée à l'origine par des nationalistes antirépublicains et xénophobes, opposés à la naturalisation des ouvriers immigrés, est désormais largement banalisée. Par la suite, Noiriel a complété ses analyses en passant des discours scientifiques aux discours politiques : il a alors mis en évidence la façon dont les contraintes liées à la « démo-

28. C. Laborde, Critical Republicanism : op. cit., p. 246 [Notre traduction].

29. Titre du premier chapitre du Creuset français.

30. G. Noiriel, Le creuset français. Histoire de l'immigration française au XIXe et XXe siècles, Paris, Seuil, 1988, p. 47.

31. Noiriel fait remarquer que Fernand Braudel, dans son œuvre magistrale sur 1'Identité de la France, ne consacre que quelques pages à l'immigration. Voir G. Noiriel, État, nation et immigration, Paris, Belin, 2001, p. 92 et sq. Pour une analyse, voir S. Guérard de Latour, Vers la république des différences, Toulouse, Presses Universitaires du Mirail, 2009, p. 125 et sq. 
cratie du public » ont contribué à la construction du "problème » de l'immigration. Ce thème a été en effet largement exploité par les partis politiques et les médias de masse pour susciter l'adhésion des classes populaires au régime républicain et construire la frontière imaginée entre les nationaux et les étrangers, frontière renforcée par la mise en place de la protection sociale à la suite de la Grande Dépression. Parmi les forces politiques en présence, c'est toutefois « la droite nationalesécuritaire » qui a le plus instrumentalisé le thème de l'immigration, d'après Noiriel, les élites bourgeoises y trouvant une façon respectable de jeter la suspicion sur les nouvelles « classes dangereuses 》- à savoir la classe ouvrière où les immigrés étaient surreprésentés - et de neutraliser les revendications de justice sociale ${ }^{32}$.

L'analyse historique du «non-lieu de mémoire », reprise dans une perspective néo-républicaine, invite donc à être sensible aux situations de domination que génère la représentation biaisée de l'identité nationale. Si celle-ci n'accorde pas, comme dans le cas de la France, de place légitime aux immigrés et à leurs descendants, elle risque d'encourager l'ethnicisation des rapports sociaux, en imposant dans l'imaginaire collectif la frontière symbolique entre les Français de souche et les autres. Un tel processus tend à constituer ce que Pettit appelle des « classes de vulnérabilité » ${ }^{33}$ : lorsque des individus sont automatiquement identifiés comme les membres d'un groupe ethnique minoritaire, ils sont dominés dans la mesure où l'assignation identitaire qu'ils subissent les rend tous, indifféremment, vulnérables à la possibilité d'être discriminés en raison de leur origine. La domination constitue à cet égard un tort collectif qui instaure une forme de solidarité négative entre les individus dominés. Inversement, la non-domination est un bien commun dont les membres d'un groupe jouissent collectivement, une fois qu'ils sont parvenus à neutraliser les causes de leur commune vulnérabilité.

C'est la raison pour laquelle le républicanisme critique, contrairement au républicanisme classique, encourage les mobilisations minoritaires. Lorsque des groupes de citoyens politisent leur identité ethnique ou raciale, comme ce fut le cas en 2006 avec la formation des Indigènes de la République du Conseil Représentatif des Associations Noires, ils cherchent à rendre publique l'expérience de la domination sociale qu'ils

32. G. Noiriel, À quoi sert «l'identité nationale »?, op. cit.; Immigration, antisémitisme et racisme en France., op. cit.

33. P. Pettit, Républicanisme, op. cit., p. 162. 
subissent en tant que descendant d'immigré indigène ou en tant que personne noire. L'identité collective qu'ils mettent alors en avant est avant tout le vecteur de leur mobilisation contre la domination, selon le processus combinant intériorisation et identification décrit par Pettit, et le support de la contestation de l'injustice qu'ils subissent ${ }^{34}$. Ces luttes, souligne Laborde, ne sont pas antirépublicaines comme le soutiennent la plupart des républicains classiques ${ }^{35}$, au sens où elles réclameraient la reconnaissance de communautés infranationales qui fragiliseraient l'unité du peuple français. Les membres des minorités ne souhaitent pas être reconnus dans leur différence culturelle, ils souhaitent simplement ne pas être dominés à cause des préjugés de la majorité relatifs à cette «différence ». En cela, l'égalité civique reste leur objectif politique ultime et sa réalisation passe par la modification en profondeur de l'imaginaire national.

[Le républicanisme critique] recommande non la reconnaissance de "l'autre", mais la pluralisation du "nous" qui fonde le sentiment de l'appartenance collective. Afin que l'identité majoritaire ne s'arroge pas le titre d'identité nationale par défaut, les identités majoritaires doivent être acceptées comme autant de modalités possibles de la francité. (...) L'enjeu est bien de repenser l'identité nationale elle-même comme une identité historiquement fluctuante, et considérablement enrichie par les apports des vagues d'immigration successive ${ }^{36}$.

Autrement dit, même si les luttes mémorielles s'expriment souvent dans une rhétorique accusatoire, qui confronte la nation à ses crimes passés, comme l'esclavage et la colonisation, elles visent moins à rejeter l'identité nationale qu'à la fonder sur de nouvelles bases. On peut à ce titre les inclure dans le nationalisme culturel, envisagé comme un processus dynamique, et considérer qu'elles participent à leur façon à la régénération morale de la nation que ce type de nationalisme prétend opérer.

34. Ce processus de mobilisation, chez Pettit, est indissociable du rôle central qu'il attribue à la contestation dans sa théorisation de la légitimité démocratique (celle-ci n'étant plus déduite du consentement à des principes de justices de vue établis a priori, comme chez les libéraux rawlsiens, mais des possibilités de recours contre les diverses formes de domination que les institutions offrent aux citoyens.

35. J. Lindgaard, «L'appel des Indigènes de la République: Contestation ou consécration du dogme républicain. Étude de cas », V. Bourdeau, R. Merrill (dir.), La république et ses démons, Paris, Ère, 2007 ; D. Schnapper, «La république face aux communautarismes ", Études, février 2004.

36. C. Laborde, Français !, op. cit., p. 122-123. 
La façon dont le républicanisme critique s'approprie la question de l'identité nationale me paraît extrêmement utile pour éviter deux écueils, ceux d'une réduction culturaliste ou d'une réduction économique des mobilisations minoritaires. Dans le premier cas, l'erreur est d'envisager ces mobilisations comme des formes de repli communautaire qui encouragent la désaffection civique. Schnapper, par exemple, invite à se méfier des "passions ethniques " parce qu'en cultivant les particularismes culturels, elles mineraient d'après elle l'attractivité de la culture nationale et du projet politique qu'elle exprime. La sociologue interprète ainsi les mobilisations ethniques dans les termes de l'opposition durkheimienne comme une forme de résurgence du « mécanique » dans « l'organique », et donc comme le symptôme d'un défaut d'intégration dans la communauté des citoyens ${ }^{37}$. Le républicanisme critique engage, au contraire, à ne pas envisager les minorités ethniques comme des groupes culturels tendant à faire sécession, mais plutôt comme des groupes ayant intériorisé les principes politiques de la république et réclamant, au nom de ces mêmes principes, un traitement plus égalitaire. Leur mobilisation n'exprimerait donc pas un rejet de l'intégration mais l'exigence d'une intégration plus équitable.

Dans le second cas, l'erreur est de négliger la dimension culturelle de la contestation minoritaire, en vertu du caractère principalement économique de l'injustice sociale. Dans cette perspective, l'ethnicisation de certains groupes procèderait avant tout de la stratification sociale et n'aurait qu'un statut dérivé. Il conviendrait ainsi de se méfier de tout discours à caractère identitaire au motif qu'il détourne l'attention des vrais problèmes, ce qui vaut tant pour les minoritaires lorsqu'ils exigent la reconnaissance que pour les majoritaires lorsqu'ils politisent la question de l'identité nationale. Contre cette vision strictement économique des problèmes sociaux, le républicanisme critique offre une vision plus nuancée des enjeux de reconnaissance qui s'apparente à l'approche de la justice sociale chez Nancy Fraser ${ }^{38}$. Celle-ci prend au sérieux le caractère mixte des injustices, lesquelles procèdent à la fois d'une distribution

37. « [Durkheim] n'a pas analysé le rapport entre les deux formes de solidarité en termes de dialectique ou de tension ni envisagé le retour possible des solidarités et des identités particulières ; il n'a pas prévu que l'intégration "mécanique" pourrait se développer à nouveau aux dépens de "l'organique" ou que les passions ethniques pourraient se ranimer aux dépens du principe civique. (D. Schnapper, La relation à l'autre, op.cit., p. 398).

38. N. Fraser, Qu'est-ce que la justice sociale? Reconnaissance et redistribution, Paris, La Découverte, 2011. 
inique des ressources économiques et des formes d'exclusion générées par les normes culturelles dominantes. Dans le cas des minorités ethniques françaises, on a vu précédemment comment la conception exclusive des «modalités de la francité » pouvait générer des formes larvées de domination. Nier cette double dimension, en arguant du caractère exclusivement économique des injustices, peut donc s'avérer politiquement conservateur : cela conduit à négliger une partie de l'expérience de la domination que vivent les minoritaires et à renforcer, faute de les combattre, les préjugés culturels de la majorité.

Le grand intérêt de l'approche de Fraser consiste à ne pas évacuer les enjeux de reconnaissance des questions de justice, tout en précisant leur statut normatif. L'idée essentielle qu'elle défend, suivie en cela par Laborde, est que la reconnaissance n'est pas une norme indépendante, mais qu'elle est dérivée d'une exigence de justice politique. Dans les termes de Fraser, c'est la «parité de participation $»^{39}$ et non l'identité personnelle ou culturelle qui justifie les demandes de reconnaissances (ainsi que le soutiennent A. Honneth ou C. Taylor). Ce qui est contestable dans le déni de reconnaissance, ce ne sont pas ses effets délétères sur la psychologie de l'individu mais le fait que le statut de citoyen égal est refusé à certains. Une reconnaissance fondée sur la "parité de participation » implique selon Fraser deux types de conditions, une « condition objective » qui « bannit les formes d'inégalité matérielle et de dépendance économique » et une " condition intersubjective » qui « suppose que les modèles institutionnalisés d'interprétation et d'évaluation expriment un égal respect pour tous les participants et assurent l'égalité des chances dans la recherche de l'estime sociale ${ }^{40}$. L'argument de Laborde à propos de la pluralisation de l'identité nationale relève de cette seconde condition : ce que contestent les minoritaires dans les représentations collectives associées à l'identité nationale, c'est qu'elles instaurent un modèle institutionnalisé de valeurs qui dénie à certaines personnes le statut de partenaires à part entière dans l'interaction sociale. La reconnaissance exigée n'a dès lors qu'une valeur instrumentale et provisoire : il s'agit de promouvoir de façon volontariste la participation des minorités aux institutions sociales et politiques afin, non pas d'accentuer la perception des différences, mais de la banaliser. L'objectif est d'écarter une vision homogène et stéréotypée de l'identité nationale pour l'associer à la diversité des façons

39. N. Fraser, Qu'est-ce que la justice sociale? op. cit., p. 52 sq.

40. Ibid., p. 54. 
d'être français. À ce titre, la pluralisation de l'identité nationale, bien qu'elle passe par la reconnaissance de la diversité ethnoculturelle de la population française, doit paradoxalement conduire à la « des-ethnicisation » des relations sociales, selon l'expression de Laborde.

\section{Troisième partie : l'ethos national et multiculturel, envisagé comme un quasi-perfectionnisme identitaire}

La première partie a permis d'établir que le républicanisme ne peut pas évacuer la question de l'identité nationale, malgré les dérives auxquelles sa politisation risque de conduire. La deuxième partie a précisé la façon critique dont le néo-républicanisme réinvestit cette question, afin de promouvoir l'intégration des minorités. L'identité nationale y est pensée comme la condition intersubjective de l'égalité civique, mais elle ne peut jouer ce rôle qu'à condition de se pluraliser et de neutraliser l'ethnicisation des rapports sociaux. Je voudrai à présent aller plus loin dans l'analyse de cette condition intersubjective, afin de préciser l'originalité du républicanisme critique par rapport aux variantes libérales du nationalisme. Certains libéraux soutiennent en effet que la portée universelle de la citoyenneté moderne n'invalide pas la valeur morale des cultures nationales. Celles-ci constituent d'après eux des contextes indispensables d'une part, pour donner du sens à l'existence individuelle et d'autre part pour forger la solidarité politique. Ces penseurs admettent donc la valeur des communautés nationales tout en se réclamant du libéralisme dans la mesure où ils proposent une défense strictement instrumentale de ces liens communautaires, qu'ils voient comme le substrat des droits individuels ${ }^{41}$ : la culture nationale est justifiée, non en vertu de sa valeur intrinsèque, mais comme « contexte de choix » (Kymlicka) nécessaire à l'exercice de l'autonomie individuelle ou comme source de motivation indispensable à la mise en œuvre de la justice distributive.

Dans ce qui suit, je voudrais montrer que le républicanisme critique invite à aller plus loin qu'une simple défense instrumentale de la culture nationale. Celle-ci doit plutôt être envisagée comme un bien commun,

41. L'exposé de cette justification libérale de l'identité nationale est très clairement exposée par Will Kymlicka dans son chapitre «Liberal Egalitarianism and Civic Republicanism : Friends or Enemies? », in Politics in the Vernacular, Oxford, Oxford University Press, 2001, p. 327-346. 
doté d'une valeur propre, pour autant qu'elle reflète la diversité culturelle des membres de la nation. Pour établir cela, je propose d'examiner la façon dont Laborde situe le républicanisme critique dans le débat qui oppose les "libéraux compréhensifs » aux « libéraux politiques ». Après avoir rappelé ce point, je tâcherai de montrer que ce qu'elle soutient à propos du principe républicain de « liberté » peut être étendu au principe républicain de la « solidarité ». Laborde n'opère pas elle-même une telle extension et reste assez proche de la position des nationalistes libéraux en ce qui concerne l'identité nationale. Or il me semble que cette proximité rend l'originalité de l'alternative républicaine au libéralisme moins évidente et que, par ailleurs, elle reflète un certain inachèvement de l'argumentation du républicanisme critique sur ce point.

Pour Laborde, en ce qui concerne la question de la liberté ou de l'autonomie, le républicanisme critique doit éviter deux écueils : d'abord celui du libéralisme compréhensif qui, parce qu'il accorde une valeur intrinsèque à la liberté individuelle, risque de verser dans le perfectionnisme politique ; ensuite celui du libéralisme politique qui, parce qu'il refuse d'écarter les cultures illibérales (quoique raisonnables) du consensus par recoupement, ne permet pas de combattre efficacement des formes de domination intériorisées dans la sphère domestique.

Comme Rawls, Laborde considère que le libéralisme compréhensif est contestable dans des sociétés marquées par le «fait du pluralisme ». De même que Rawls, en libéral, conteste l'usage abusif du pouvoir que fait une majorité libérale lorsqu'elle érige sa vérité morale en « doctrine sectaire » ${ }^{42}$, Laborde, en néo-républicaine, comme rejette l'imperium, à savoir l'abus de pouvoir commis par l'État lorsqu'il interfère arbitrairement dans la vie des citoyens pour les forcer à se conformer à un idéal moral ${ }^{43}$. Rappelons que la conception néo-républicaine de la liberté reste négative : la non-domination n'implique pas d'idéal substantiel de vertu et s'avère compatible avec une grande diversité de façons de vivre, même celles qu'adoptent les religieux traditionnalistes ${ }^{44}$.

42. J. Rawls, «Justice as Fairness : Political not Metaphysical », Philosophy and Public Affairs, 14 (3), 1985, p. 246.

43. C. Laborde, «Female Autonomy, Education and the Hijab», Critical Review of International Social and Political Philosophy, 9 (3), 2006, p. 351-377.

44. J. Rawls, « Justice as Fairness : Political not Metaphysical », art. cité ; P. Pettit, Républicanisme, op. cit., p. 130 «La non-domination et l'État moderne et pluraliste». 
Mais Laborde reproche au libéralisme politique de ne pas suffisamment prendre au sérieux l'autre source de domination, le dominium, qui s'instaure dans la vie sociale du fait de certaines représentations/pratiques religieuses et culturelles. Il ne suffit pas, par exemple, d'invoquer la liberté de conscience pour justifier des pratiques religieuses à caractère patriarcal et sexiste. Dans de tels cas, un néo-républicain se doit de rester vigilant face aux formes insidieuses de domination qui se mettent en place lorsque les enfants - et tout particulièrement les jeunes filles - intériorisent les attentes de leur groupe social et culturel. Les rapports de domination naissent précisément de ces processus d'adaptation des préférences, qui conduit un individu à modeler ses choix de vie sur le statut inégal qui lui est attribué.

Laborde propose ainsi une voie intermédiaire qui repose sur une asymétrie essentielle entre la sphère éducative et la sphère sociale : dans la première, il convient dit-elle de défendre une conception "plutôt compréhensive » de l'autonomie, en inculquant aux enfants un ethos marqué par l'autonomie personnelle, c'est-à-dire en les entraînant à développer certaines dispositions (telles que l'esprit critique, la confiance en soi, la tolérance, la connaissance de ses droits). Dans la seconde, en revanche, l'État doit respecter les choix des citoyens, même si ces choix rompent avec le mode de vie moderne et libéré, comme dans le cas des intégristes religieux. L'asymétrie vise ici à respecter le fait du pluralisme tout en créant les conditions d'un pluralisme non dominant : les citoyens d'une république moderne doivent admettre une grande pluralité de modes de vie, même ceux qui semblent rejeter la valeur de l'autonomie personnelle, à condition qu'ils soient assurés que ceux-ci ne résultent pas de préférences adaptatives encourageant des formes de servitude volontaire. Une telle position peut être qualifiée de « quasi-perfectionniste » ${ }^{45}$, selon l'expression du néo-républicain John Maynor, dans la mesure où elle présente l'autonomie comme un type de perfection que doit atteindre la personne dans sa formation morale : il est légitime de promouvoir la valeur morale de l'autonomie dans l'éducation des futurs citoyens, dans la mesure où cette valeur leur donne accès à une vie meilleure, plus riche, en ce qu'elle les prépare à agir, une fois adulte, d'une façon qui n'encourage pas les rapports de domination. Toutefois, l'acquisition de cette vertu reste compatible avec une

45. J. Maynor, Republicanism and the Modern World, Cambridge, U.K., Polity ; Malden, MA, 2003, p. 80. 
grande variété de modes de vie, lesquels peuvent accorder à l'autonomie personnelle une place secondaire dans leur vie d'adultes.

Ceci étant posé, je voudrai soutenir que cette position quasi-perfectionniste ne s'applique pas seulement à la question de la liberté républicaine, mais aussi à celle de la solidarité républicaine, autrement dit qu'elle a des implications non seulement relativement aux valeurs partagées mais aussi relativement à l'identité commune. Plus précisément, je soutiendrai la chose suivante : de même que, du point de vue du républicanisme critique, il faut accorder une valeur substantielle à l'autonomie personnelle dans l'éducation civique, afin de créer les conditions sociales et politiques d'une forme de pluralisme exempte de rapports de domination, il convient d'accorder une valeur substantielle à la conception multiculturelle de l'identité nationale, si l'on veut jeter les bases d'une solidarité politique réellement inclusive.

Il s'agit donc ici de reproduire l'asymétrie soulignée par Laborde dans le cas de l'autonomie en l'appliquant au cas de l'identité nationale. Dans une conception quasi-perfectionniste du républicanisme, les citoyens adultes ne devraient être contraints ni d'adopter des modes de vie libérés, ni de chérir une identité politique multiculturelle; tout comme ils doivent rester libres d'être traditionnalistes et conservateurs, ils doivent rester libres de privilégier le type d'identité culturelle qu'ils préfèrent, voire de n'en cultiver aucune en particulier. Toutefois, de tels choix identitaires ne seront compatibles les uns avec les autres qu'à condition d'être contraints, à la base, par l'ethos national et multiculturel qu'on aura inculqué aux futurs citoyens. Si les citoyens ne partagent pas d'emblée la croyance que l'on peut être, par exemple, authentiquement français et d'origine immigrée, le risque est d'encourager la suspicion des citoyens issus de la culture majoritaire à l'égard des manifestations publiques des cultures issues de l'immigration et la lecture de tels comportements comme des symptômes de "distance culturelle », de "problème d'intégration» à la nation française. Réciproquement, les citoyens issus de l'immigration tendront à intérioriser les stéréotypes de la majorité relativement à leur " étrangeté » supposée. Dans les deux cas, la vision réductrice de l'identité nationale confortera des rapports de domination. Cela suggère, a contrario, que la capacité offerte aux citoyens de cultiver les références culturelles de leur choix, sans être de ce fait soumis à des préjugés racistes et ethnocentriques, dépend de l'intériorisation préalable d'une identité nationale représentative de cette diversité. 
Cette position permet de préciser un point qui reste ambigu chez Laborde. D'un côté, celle-ci critique les politiques volontaristes de reconnaissance auxquelles elle reproche de renforcer les préjugés ethniques qu'elles prétendent combattre ${ }^{46}$. D'un autre côté, elle défend pourtant l'idée qu'on peut en faire un usage stratégique afin de banaliser les identités minoritaires. Précisons toutefois que Laborde n'applique explicitement ce type de stratégie qu'à la sphère des médias (la sphère de l'éducation devant rester indifférente aux identités ethniques, et la sphère économique devant se limiter à une " politique de lutte agressive contre les discriminations »). En effet, dans la mesure où les médias jouent un rôle crucial dans la formation des représentations collectives et où le choix des acteurs médiatiques repose sur une multitude de critères non réductibles au mérite (en particulier sur leur apparence physique), il n'est pas illégitime d'après elle d'y promouvoir activement la présence de minorités visibles afin de pluraliser l'imaginaire national. Seulement, je considère qu'il ne suffit pas de justifier cette concession à la logique de la reconnaissance par des raisons stratégiques pour conjurer le risque de l'essentialisation : imposer politiquement la reconnaissance de ce type de diversité, même dans la seule sphère des médias, risque d'être perçu comme une injonction arbitraire et illégitime par les membres de la majorité qui ne se reconnaissent pas dans ces références culturelles, notamment parce que certains ont déjà intériorisé une vision stéréotypée et exclusive de l'identité nationale.

C'est pour éviter une telle ambiguïté que je propose d'appliquer à la question de l'identité nationale la structure asymétrique que Laborde mobilise en vue de déterminer la juste place de la valeur d'autonomie. Pour que la reconnaissance positive de la diversité culturelle ne soit pas perçue comme une forme de politique arbitraire aux effets souvent contre-productifs, il convient de la mettre en œuvre avant tout et principalement dans la sphère éducative. C'est là qu'il faut promouvoir activement un ethos national et multiculturel, fondé sur l'inculcation d'un imaginaire collectif pluralisé. Il faut souligner ici que la structure asymétrique invite à dépasser le cadre d'une justification strictement instrumentale de la culture nationale que Laborde maintient, à la suite des nationalistes libéraux. En effet, de même que l'éducation à l'autonomie repose sur la conviction que celle-ci a une valeur «plutôt

46. « Critical republicanism therefore opts for "strategies of de-ethnicization" which "sees politics as a way of resisting, rather than recognizing particularist identification » (C. Laborde, Critical Republicanism, op. cit., p. 238). 
compréhensive ", l'éducation à la solidarité repose sur une valorisation substantielle de la culture nationale. Cette thèse peut surprendre, dans la mesure où, s'il y a de bonnes raisons de considérer l'autonomie comme une valeur substantielle dans le cadre de la citoyenneté républicaine (laquelle s'est toujours réclamée d'un idéal de liberté), il n'en va pas de même avec l'identité nationale. Celle-ci manque apparemment du contenu normatif que possède l'autonomie. Elle relève des faits plutôt de que des valeurs, son lien avec la citoyenneté semblant s'ancrer sur des circonstances historiques contingentes. Or, je considère que cette façon de déprécier le lien national procède de la confusion souvent faite entre le nationalisme ethnique et le nationalisme culturel. Cette confusion tend à envisager chaque culture nationale moderne comme la forme généralisée et dominante d'une identité ethnique qui aurait préexisté aux institutions démocratiques. L'intuition des républicains à propos du patriotisme invite au contraire à envisager les identités nationales, non comme des données pré-politiques mais comme des formes culturelles inédites de solidarité, produites par le dispositif institutionnel démocratique. Les identités nationales démocratiques sont à cet égard la manifestation d'une nouvelle possibilité sociologique, celle d'une identification collective à la fois égalitaire et pluraliste, capable de ménager une place à la diversité ethnoculturelle.

Pour illustrer ce point, on peut revenir à l'analyse du nationalisme culturel français. Lorsque la réaction romantique allemande a obligé les élites intellectuelles françaises à tracer les contours identitaires du peuple français, historiens et géographes n'ont pas érigé en norme une culture officielle homogène et monolithique, ils ont au contraire ménagé une place importante à la diversité régionale. On peut évoquer le cas des " esprits frontaliers» dans l'Histoire de France de Michelet qui décrit la capacité de la personnalité française à se décliner au pluriel, au contact d'autres cultures nationales ${ }^{47}$. On peut aussi évoquer la diversité des terroirs mise en avant par Paul Vidal de la Blache dans son Tableau de la géographie de la France ${ }^{48}$. Il est bon de rappeler que cette conception pluraliste de la culture nationale ne s'est pas cantonnée aux discours savants, mais qu'elle a été largement diffusée au sein de la population, tout particulièrement par le biais de l'éducation nationale. On pensera ici au succès du manuel républicain d'Augustine Fouillé, Le tour de la

47. J. Michelet, Le tableau de la France, Paris, Orban, 1987 (1 re éd. 1832).

48. P. Vidal de la Blache, Tableau de la géographie de la France, Paris, Hachette, 1908 (1 re éd., 1903). 
France par deux enfants qui trouva dans la géographie un vecteur puissant d'initiation et de sensibilisation des petits Français à la richesse de leur territoire national ${ }^{49}$. L'assimilation culturelle orchestrée par la III République ne se confond donc pas, comme on l'affirme souvent, avec l'effacement des différences. La diversité culturelle constitue au contraire l'un des traits distinctifs de l'identité nationale, qui se manifeste encore de nos jours à travers l'attachement fort des Français à leurs terroirs. Or, si cette synthèse entre unité nationale et diversité culturelle est possible dans le cas des identités régionales, pourquoi ne le serait-elle pas dans le cas d'autres origines ethniques? Autrement dit, s'il est tout à fait admis dans le sens commun français que l'on peut être franco-breton, pourquoi ne pas admettre avec la même évidence que l'on peut être franco-algérien?

Bien évidemment, on réagira immédiatement à cette proposition en faisant valoir la distance qui sépare une identité régionale et folklorique (dans le cas de la Bretagne) et une identité politique, chargée d'un lourd passé de domination coloniale et d'affrontements politiques (dans le cas de l'Algérie). Ce qui rend difficilement envisageable, à l'heure actuelle, la citoyenneté à trait d'union franco-algérienne, c'est précisément que la lecture politique de l'identité algérienne reste prégnante dans les esprits et qu'elle empêche précisément sa folklorisation. La mise en avant de cette identité (et des autres origines post-coloniales) est dès lors perçue comme un risque politique, comme un signe d'hostilité à l'égard de la république française. Il ne s'agit pas de statuer sur la réalité de cette hostilité, mais seulement de souligner que, même si elle existe dans certains cas, elle n'empêche pas, en tant que telle, la synthèse entre l'identité nationale et les origines extra-nationales. Je rappellerai ici ce qui est bien connu, à savoir que les " petites nations » ne se sont pas toutes fondues de bonne grâce dans le creuset républicain et que la méfiance à l'égard de certaines cultures régionales a pu susciter autant de méfiance en son temps que d'autres cultures étrangères. Michelet lui-même, malgré le portait pluraliste qu'il brossât de la France dans son Histoire de France, insista sur l'urgence à faire valoir l'esprit universaliste et progressiste du centre de la France (la capitale) contre les forces traditionnalistes et conservatrices de la périphérie (les régions). Le précédent de la fusion des identités régionales dans l'imagi-

49. G. Bruno (A. Fouillée), Le Tour de la France par deux enfants. Devoir et patrie. Livre de lecture courante avec 200 gravures instructives pour leçon de choses, Paris, Belin, 1877. 
naire national suggère donc la possibilité de nouvelles synthèses culturelles : lorsque les identités minoritaires ne fonctionnent plus comme des stigmates sociaux et que leur charge politique est neutralisée, rien n'empêche les différences culturelles dont elles sont porteuses d'enrichir les cadres identitaires de la nation. L'enjeu est bien, comme le dit Laborde, de banaliser les identités minoritaires, mais ce que le cas des identités régionales suggère plus précisément qu'elle ne le dit, c'est que cette banalisation est étroitement liée à l'intériorisation d'un imaginaire national, où domine l'idée que la diversité culturelle constitue une richesse propre du pays. Or dans une telle perspective, on accorde bien à l'identité nationale une valeur substantielle qui ne saurait se réduire à une valeur strictement instrumentale.

\section{Conclusion}

$\mathrm{Au}$ terme de cette analyse, il semble donc que le républicanisme critique soit porteur d'une conception originale du rôle de l'identité nationale. Contrairement aux nationalistes libéraux qui envisagent les cultures nationales comme des données pré-politiques, avec lesquelles l'universalisme civique doit composer, les républicains critiquent envisagent l'identité nationale non seulement comme un effet des institutions démocratiques mais aussi comme la condition de possibilité de leur bon fonctionnement. La place des minorités n'y est donc pas pensée de la même manière ; encore une fois, leur culture n'est pas envisagée comme une donnée que certains droits, dits «culturels» viendraient garantir contre les velléités assimilationnistes de la culture ethnique majoritaire, mais comme une source de diversité susceptible d'enrichir l'imaginaire national d'une communauté moderne de citoyens. Autrement dit, dans la perspective républicaine, la justice ne doit pas permettre la cohabitation de plusieurs groupes culturels; elle doit plutôt instituer l'élaboration d'une culture commune et dynamique, capable d'évoluer avec sa population. J'ai tâché de soutenir, dans le dernier moment de ce texte, que le caractère à la fois commun et diversifié de cette culture devrait être intériorisé au plus tôt, par une politique volontariste en matière d'éducation civique. À ce stade de la formation individuelle, une certaine dose de perfectionnisme reste légitime ; il permet dans ce cas de promouvoir l'identité nationale et multiculturelle comme un bien commun auquel les futurs citoyens doivent être atta- 
chés, mais à l'égard duquel ils pourront, en tant que citoyens adultes, prendre une certaine distance. Par la reprise de cette structure asymétrique, j'ai cherché à éviter les écueils d'une politique volontariste de la reconnaissance qui, appliquée indifféremment à toutes les sphères sociales, risquent de renforcer les préjugés racistes ou ethnocentriques qu'elle prétend combattre.

\section{Bibliographie}

Anderson B., L'imaginaire national, Paris, La Découverte, 2002.

Bruno G, (Fouillée, Augustine), Le Tour de la France par deux enfants.

Devoir et patrie. Livre de lecture courante avec 200 gravures instructives pour leçon de choses, Paris, Belin, 1877.

FRASER N., Qu'est-ce que la justice sociale? Reconnaissance et redistribution, Paris, La Découverte, 2011.

GuÉrard de Latour S., Vers la république des differences, Toulouse, Presses Universitaires du Mirail, 2009.

-, « Reworking the neo-republican sense of belonging », Diacritica, 24/2, 2010.

Hutchinson J., « Re-interpreting Cultural Nationalism », Australian Journal of Politics and History, 45/3, 1999.

Kymlicka W., «Liberal Egalitarianism and Civic Republicanism : Friends or Enemies? », Politics in the Vernacular, Oxford, Oxford University Press, 2001.

-, La citoyenneté multiculturelle. Une défense libérale du droit des minorités, Paris, La Découverte, 1995.

Laborde C., « Female Autonomy, Education and the Hijab », Critical Review of International Social and Political Philosophy, 9 (3), 2006.

-, Critical Republicanism. The Hijab Controversy and Political Theory, Oxford, Oxford University Press, 2008.

-, Français, encore un effort pour être républicains!, Paris, Le Seuil, 2010.

LindGaARD J., «L'appel des Indigènes de la République : Contestation ou consécration du dogme républicain. étude de cas ", in V. Bourdeau, R. Merrill (dir.), La république et ses démons, Paris, Ère, 2007.

Martigny V., «The Importance of Culture in Civic Nations : Culture and the Republic in France ", Studies in Ethnicity and Nationalism, vol. 8, $\mathrm{n}^{\circ} 3,2008$.

MaYnor J., Republicanism and the Modern World, Cambridge, U.K., Polity ; Malden, MA, 2003.

Michelet J., Le tableau de la France, Paris, Orban, 1987. 
NoIRIEL G., Le creuset français. Histoire de l'immigration française au $\mathrm{XIX}^{e}$ et $\mathrm{xx}^{e}$ siècles, Paris, Le Seuil, 1988.

-, État, nation et immigration, Paris, Belin, 2001.

-, À quoi sert « l'identité nationale », Paris, Agone, 2007.

-, Immigration, antisémitisme et racisme en France. Discours publics, humiliations privées (XIX ${ }^{e}-\mathrm{XX}^{e}$ siècles), Paris, Fayard, 2007.

Petтit P., Républicanisme. Une théorie de la liberté et du gouvernement, Paris, Gallimard, 1997.

-, « Option-Freedom and Agency-Freedom », Journal of Theoretical Politics, 15(4), 2003.

Rawls J., « Justice as Fairness : Political not Metaphysical », Philosophy and Public Affairs, 14 (3), 1985.

Rousseau J.-J., Contrat social, Paris, G.-F., 1996.

SCHNAPPER D., La relation à l'autre. Au cour de la pensée sociologique, Paris, Gallimard, 1998.

-, « La république face aux communautarismes », Études, février 2004.

SKInNER Q., La liberté avant le libéralisme, Paris, Le Seuil, « Liber », 2000.

TAMIR Y., Liberal Nationalism, Princeton, Princeton University Press, 1990.

VAlluy J.(dir.), «Xénophobie de gouvernement, nationalisme d'État », Cultures \& Conflits, ${ }^{\circ} 69,2008$.

Vidal de la Blache P., Tableau de la géographie de la France, Paris, Hachette, 1908.

VIroli M., For the Love of Country. Essays on Patriotism and Nationalism, Oxford, Oxford University Press, 1997. 